\title{
Le territoire innovant
}

\section{Innovative territory}

\author{
Corinne Tanguy ${ }^{1}$ \\ ${ }^{1}$ AgroSup Dijon, Laboratoire CESAER (AgroSup Dijon, INRA, Univ. Bourgogne Franche-Comté, F-21000 Dijon, France), \\ Réseau de Recherche sur l'Innovation (RRI), corinne.tanguy@inra.fr
}

RÉSUMÉ. La littérature économique consacrée aux systèmes territoriaux d'innovation (district, milieu innovateur, cluster, etc.) met l'accent sur le rôle fondamental du territoire et de la proximité dans les collaborations entre acteurs. L'article propose de développer un regard critique sur les clusters (et les notions proches) et de discuter le rôle joué par la proximité géographique. Nous mettons en particulier en évidence l'importance des relations à distance permises par les mobilités et les techniques de communication ainsi que la manière dont la combinaison de différentes formes de proximité (géographique, organisée, relationnelle, cognitive, sociale) constitue potentiellement des conditions propices à l'apprentissage, à la constitution de réseaux et par conséquent à l'innovation, inscrites dans et révélées par le territoire.

ABSTRACT. Research on regional innovation systems has highlighted the crucial role of territory and the effects of proximity in the development of cooperative relations between actors. In this article we develop a critical analysis of clusters and similar notions and we discuss the role of geographical proximity. The importance of distant relationships enabled by increasing mobility and information technology are explored. We analyze how a combination of different forms of proximity (geographic, organized, relational, cognitive and social) potentially foster learning and networking. This in turn leads to innovation inscribed in and revealed at the territory level.

MOTS-CLÉS. Proximités, réseaux d'innovation, coopérations, cluster, territoires de projet.

KEYWORDS. Proximities, innovation networks, cooperation, cluster, project territories.

L'analyse des processus d'innovation et des liens qu'ils entretiennent avec les aspects territoriaux est une thématique de recherche importante et un enjeu en termes de politiques publiques, comme l'illustrent les publications scientifiques et le nombre de rapports récents ayant trait au sujet [MAD, 08, GOD, 10, SCH, 14, CMI, 15, OUR, 15]. Géographie de l'innovation, districts technologiques, milieux innovateurs, clusters d'innovation, technopoles, ..., les termes sont nombreux, correspondant souvent à des visions différentes du processus d'innovation et de son inscription spatiale. Mais au-delà de leurs divergences, ces approches présentent un point commun : elles considèrent que l'innovation peut posséder une dimension spatiale forte et que les firmes retirent un avantage à se localiser dans le même espace [AUD,96]. La disponibilité de ressources financières, d'un capital social et relationnel, l'existence de services support ou la présence d'universités et centres de recherche sur un territoire sont des éléments clés explicatifs de la capacité des acteurs à innover. La proximité géographique constitue alors le ferment principal de regroupement des firmes au niveau régional ou local.

La création d'entreprises innovantes est également un leitmotiv des politiques de développement économique local avec comme modèle les exemples de la Silicon Valley et autres «clusters » d'entreprises, appartenant le plus souvent à des secteurs intensifs en connaissances. Ainsi, le rapport sur la créativité et l'innovation dans les territoires [GOD, 10] cherche-t-il à identifier de nouvelles formes de créativité et d'innovation en mesure d'améliorer les deux dimensions du développement des territoires que sont sa compétitivité et son attractivité. Dans cette perspective, la définition de l'innovation proposée est une définition élargie qui ne se limite pas aux innovations technologiques de produit ou de procédé s'appuyant sur de la Recherche et Développement. L'innovation dont il sera question est tout autant sociale, territoriale, organisationnelle ou technologique.

Cette contribution propose de poser un regard critique sur les débats et enjeux qui se posent aujourd'hui dans le champ de «l'innovation ouverte » [CHE, 03], qui postule la nécessaire ouverture de l'entreprise innovante sur son territoire et sur les sources de connaissances externes. Quels facteurs 
organisationnels, économiques et territoriaux déterminent dans ce cas ces coopérations ? Comment définir le territoire innovant ou territoire de coopération et «d'absorption » de ressources externes des entreprises?

Pour ce faire, nous verrons dans un premier temps que la littérature économique consacrée aux systèmes territoriaux d'innovation (districts, milieux innovateurs, clusters, etc.) met l'accent sur le rôle fondamental du territoire et de la proximité dans les collaborations entre acteurs. Notre objectif dans un second temps sera de développer un regard critique sur les clusters (et les notions proches) et de discuter le rôle joué par la proximité géographique. En effet il est important de prendre en compte les relations à distance permises par les mobilités et les techniques de communication, ainsi que la manière dont la combinaison de différentes formes de proximités (géographique, relationnelle, organisée, ...) constitue des conditions propices à l'apprentissage, à la constitution de réseaux et par conséquent à l'innovation, inscrites dans et révélées par le territoire. En conclusion, nous mettrons l'accent sur le développement récent d'innovations en mesure de favoriser une transition écologique de nos sociétés. Elle se traduit aujourd'hui par la construction de nouveaux territoires de projets avec des objectifs d'économies en termes d'approvisionnement d'énergie, de ressources, de traitement de déchets, ou encore de rendements d'échelle générés par les mutualisations de services [TOR,15]. Dans cette perspective, la question de la coordination des différentes parties prenantes sur un territoire et des innovations environnementales et sociales est prépondérante.

\section{Territoire et Innovation : un processus collectif de co-construction}

Le territoire a longtemps été considéré en économie comme une variable d'ajustement sans existence propre. Schumpeter a mis très tôt l'accent sur l'apparition en grappe des innovations ou les regroupements d'entrepreneurs innovateurs, mais la question de l'espace n'était pas présente dans ses analyses. Perroux [PER, 64], quant lui, s'est focalisé dans son analyse des pôles de croissance sur le rôle des grandes entreprises en mesure d'entraîner la croissance de leur environnement économique.

À partir des années 1980, différents travaux dont ceux du GREMI (Groupe de Recherche Européen sur les Milieux Innovateurs) [AYD, 86, MOR, 98, CAM, 06] insistent sur l'importance de la dimension spatiale dans les processus d'innovation. Les économistes évolutionnistes, avec la notion de système national d'innovation [LUN, 92, NEL, 93], mettent également l'accent sur l'importance des règles, lois et politiques économiques différentes selon les espaces et l'inscription des processus d'innovation dans l'espace. La capacité d'une firme à «absorber» des connaissances externes, ou à capter des «spills overs » ou «externalités de connaissances » [MAS, 01, CHA, 12], est un déterminant critique de sa capacité d'innovation et une firme sera plus ou moins en mesure d'exploiter les opportunités technologiques de son environnement selon son savoir de base, le processus d'apprentissage qui s'effectue en son sein et son mode d'organisation [COH, 89, $\mathrm{COH}, 90, \mathrm{BAT}, 04]$. Dans cette perspective, le recours à l'environnement technologique est un processus complexe, incertain, cumulatif, fortement interactif, voire coopératif, d'acquisition des ressources d'innovation et de constitution d'apprentissages collectifs et la proximité des sources d'apprentissage devient une dimension à prendre en compte. En effet, la nature tacite des connaissances, difficiles à échanger à distance et fortement «encastrées » dans des organisations et collectifs [FOR,00], expliquerait l'importance des processus locaux de collaborations et d'innovation. Ces échanges de connaissances sont selon de nombreux auteurs à la base des performances des districts italiens [BEC, 79], des milieux innovateurs [CAM, 95], des systèmes nationaux et régionaux d'innovation [LUN, 92], des clusters et des pôles de compétitivité. Les tenants de ces approches postulent l'existence et le rôle primordial des interactions locales dans les regroupements d'entreprises. 


\section{Les districts industriels}

Les districts, notion présente dans les travaux d'Alfred Marshall [MAR, 19] ont été redécouverts par les économistes italiens étudiant la Troisième Italie [BRU, 82, GAR, 92]. Becattini [BEC, 90] (1990) définit le district industriel comme « une entité socio-territoriale caractérisée par la présence active à la fois d'une communauté de personnes et d'une population de firmes dans une aire naturellement et historiquement liée », qui résulte de l'articulation d'une population de firmes localisées et d'une communauté locale. Celle-ci s'appuie sur l'existence de représentations favorables à l'initiative et au développement économiques, qui s'incarnent dans un système d'institutions (le marché, la famille, l'école, l'église, ...) et de règles diffusant ces valeurs dans l'ensemble de la zone et assurant sa transmission entre générations. Dans la Troisième Italie, ce sont surtout les réseaux familiaux qui jouent ce rôle.

\section{Les systèmes de production localisés (SPL)}

Les systèmes de production localisés désignent un ensemble d'activités interdépendantes, techniquement et économiquement organisées et territorialement agglomérées. Un SPL se fonde sur une grande diversité d'acteurs locaux : des ménages, également consommateurs, ainsi que des acteurs de la production ou des personnels administratifs ; des entreprises de toutes tailles et statuts ; mais aussi des pouvoirs public et institutions locales telles que chambres de commerce ou organismes de gestion locale. Le dispositif des systèmes productifs locaux lancé par la DATAR en 1998 a été tout particulièrement orienté vers le soutien aux PME et entreprises de taille intermédiaire. Cette politique s'est traduite par un dispositif de soutien logistique et financier à des réseaux sélectionnés de firmes, dans l'objectif d'accroître leurs performances productives, d'améliorer leur gestion des ressources humaines et de favoriser le développement de l'innovation.

\section{Les milieux innovateurs}

Le GREMI (Groupe de Recherche Européen sur les Milieux Innovateurs) [AYD, 86, CAM, 06] fait l'hypothèse que les processus d'innovation reposent sur des relations de proximité géographique. Le milieu innovateur constitue le contexte approprié à leur formation et à leur développement car les agents y partagent des représentations semblables, identifient rapidement des partenaires en leur sein, échangent des informations, se font confiance... L'innovation est conçue comme un processus collectif, qui implique un ensemble de relations formelles et informelles s'exprimant au sein de réseaux d'innovation localisés composés d'acteurs (laboratoires publics, centres de recherche technique, entreprises, etc.) qui ne disposent pas à titre individuel des ressources suffisantes pour innover. Cette stratégie de coopération, qui s'organise souvent de manière durable, permet à la fois une amélioration de la créativité et une réduction des risques et des coûts inhérents au processus d'innovation [MAI, 95].

\section{Les clusters}

Porter [POR, 98, POR, 03] affirme qu'un cluster est « ... un groupe géographiquement proche de firmes et d'institutions associées, interconnectées au sein d'un champ particulier et liées par des éléments communs et des complémentarités ». Dans un premier temps, la notion de cluster s'est appliquée à des success stories, avec l'exemple emblématique de la Silicon Valley. Par la suite, la tendance a été à l'extension tous azimuts de la notion de cluster, vers des systèmes moins axés sur les activités de haute technologie ou dont le degré de performance est plus faible, mais aussi en tant qu'outil de politique économique locale ou nationale (politique des pôles de compétitivité en France). L'hypothèse est que la mise en synergie des entreprises locales s'avère toujours bénéfique, la circulation des connaissances étant nécessaire au bon fonctionnement de n'importe quel système organisé. Du coup, les limites géographiques des clusters varient fortement selon les études, de la zone d'activités innovantes jusqu'au département ou à la région.

\section{Encadré 1. Les différents systèmes territorialisés d'innovation [TOR, 14]}


Ainsi, la littérature économique consacrée aux systèmes territoriaux d'innovation (districts, milieux innovateurs, clusters, etc.) met l'accent sur le rôle fondamental du territoire et de la proximité entre cocontractants. Dans cette littérature, le territoire n'est pas considéré comme un stock de ressources génériques disponibles dans lesquelles les entreprises pourraient puiser à loisir, mais il s'agit d'un construit social résultant d'un processus de coordination des acteurs et de construction de ressources territoriales [COL, 04]. Deux dimensions apparaissent particulièrement constitutives des systèmes territoriaux : l'innovation, les apprentissages, les savoir-faire et ressources historiquement construits comme principe de compétitivité ; les réseaux comme principe organisationnel, réseaux qui reposent avant tout sur des relations de proximité géographique. Il s'agit en effet d'un contexte approprié à leur formation et à leur développement car les agents y partagent des représentations semblables, identifient rapidement des partenaires en leur sein, échangent des informations, se font confiance. Cette dimension a été renforcée depuis une quinzaine d'années dans la mise en œuvre des politiques publiques. Ainsi la politique des pôles de compétitivité mise en œuvre en France depuis 2004 a pour objectif de favoriser les projets collaboratifs de recherche et de développement entre acteurs localisés sur un même territoire [TAN, 16]. La recherche des synergies entre acteurs locaux est en effet devenue la base de la plupart des politiques de développement local.

Le succès de la notion de cluster et de ses différentes déclinaisons repose sur différentes caractéristiques des systèmes ou des réseaux d'acteurs [TOR, 06, TOR, 14] : la diffusion et circulation des connaissances au niveau local ; les externalités de réseaux, au niveau local et inter-entreprises ; la mise en commun d'infrastructures et diminution des coûts de transaction entre les participants [KAR, 05].

Depuis le début des années 1990, de nombreux travaux ont tenté de fournir une validation empirique à ces hypothèses en «mesurant » la dimension spatiale des processus d'innovation et de coopération [AUD, 96]. Ces recherches ont souligné la dimension locale des échanges de connaissances et des collaborations entre firmes. D'autres travaux ont cependant choisi de mettre l'accent sur les tendances à la globalisation de la technologie et des échanges de connaissances et sur la nécessaire articulation des dynamiques locales et globales. Ainsi dans les travaux les plus récents sur les clusters, on insiste sur le fait que les firmes ont tendance à développer plus de liens inter-clusters, donc distants géographiquement [DEP, 09]. Les clusters sont en effet des systèmes ouverts, accordant une attention particulière aux relations avec des acteurs extérieurs, ainsi qu'aux politiques nationales ou supranationales. Au-delà des phénomènes de regroupement d'acteurs localisés sur un même territoire, ce sont donc bien les phénomènes de réseaux et de collaborations établis à différentes échelles spatiales qui constituent aujourd'hui un élément central, explicatif des dynamiques d'innovation des firmes [VEL, 93, 94].

Tout en admettant ce rôle essentiel de la dimension territoriale dans les processus d'innovation, l'hypothèse de la plus grande facilité des entreprises et autres acteurs (organismes de recherche, universités, instituts techniques, etc.) à absorber les connaissances et technologies externes, lorsqu'ils se trouvent à proximité les uns des autres, a été nuancée, en particulier dans les travaux des économistes de la proximité.

\section{Proximités et réseaux de coopération territoriaux}

\subsection{La remise en cause du rôle prépondérant de la proximité géographique}

La notion de proximité non spatiale a ainsi été développée par un groupe de chercheurs français réunis dans ce qu'on appelle l'école de la proximité [BOU, 08b, BOU, 08a, RAL, 04, RER, 93, 08]. Le concept de proximité non spatiale ou organisée [TOR,05] rend compte du fait que les interlocuteurs partagent des règles communes en matière d'organisation, de représentations, de modèles et de règles d'action. Ces règles communes contribuent à faciliter les collaborations entre acteurs et leur prise en compte dans l'étude de la coordination des acteurs participe à la remise en cause du rôle prépondérant de la proximité géographique [KNO, 06]. 
D'autres facteurs expliquent ainsi la concentration géographique des acteurs et leur rôle dans la coordination des activités d'innovation et de recherche [TOR,06]. Les relations économiques sont encastrées dans des réseaux sociaux possédant souvent des bases territoriales fortes. Ainsi la coopération s'établit souvent entre des chercheurs ou des ingénieurs, issus de la même université ou appartenant au même réseau social ou familial, [GRO, 03] localisés sur le même territoire. Il ne suffit pas de réunir des acteurs au sein du même espace pour qu'ils collaborent, encore faut-il qu'ils appartiennent au même réseau ou qu'ils partagent des représentations communes. D'autre part, d'autres facteurs classiques (attractivité en matière de prix du foncier, incitations des pouvoirs publics et avantages financiers et fiscaux, marchés locaux du travail [KRU, 91] et réservoir de travailleurs qualifiés et disponibles sur le marché local) peuvent expliquer le succès des agglomérations locales, y compris en l'absence de liens forts en termes de synergie [TOR, 06, GOR, 05].

L'ensemble des travaux sur les regroupements d'acteurs présentent l'inconvénient de focaliser l'attention sur le rôle de la proximité géographique entre acteurs, alors que de nombreuses études montrent des entreprises qui développent des stratégies mêlant étroitement relations de proximité et relations plus «éloignées » [ZIM ,98, KLE, 00, MAL, 02, BOS, 05, MEN, 06]. Les besoins de proximité géographique sont à relativiser si on se réfère aux arguments suivants :

- La proximité géographique peut être impulsée par les politiques publiques de manière à faciliter le développement du territoire et conduire à de l'agglomération simple sans interactions entre les acteurs regroupés.

- Le développement constant des technologies de l'information et de la communication (TIC) [RAL, 07] permet la transmission à distance d'informations toujours plus complexes et détaillées, et favorise la circulation de connaissances informelles. Ces innovations facilitent les échanges et collaborations à distance et relativisent ce besoin de proximité ou de co-localisation.

- La proximité géographique peut aussi être temporaire et les interactions de face à face ne nécessitent pas pour les acteurs de se co-localiser de manière permanente [GAL, 04, MAS, 05, SHE, 12]. La mobilité des acteurs permet de créer de la proximité temporaire quand elle est nécessaire au cours du déroulement d'un projet [BRE, 01, TOR, 10]. Bathelt et Schuldt [BAT, 08] prennent ainsi l'exemple de l'explosion des foires et colloques qui s'avèrent propices aux interactions de face à face, nécessaires à l'établissement de relations de confiance et de coopération à certains stades des projets.

La proximité géographique peut également revêtir des effets négatifs qui ne sont pas toujours soulignés en tant que tels. Elle est généralement présentée comme systématiquement bénéfique [BEN, 13, BOS, 04, ZIM, 08]. Or, la proximité peut par exemple faciliter l'imitation de l'innovation d'une entreprise par ses concurrents directs [SUI,08], ou le recrutement de personnels très spécialisés travaillant dans une entreprise voisine, et être un facteur de désincitation à innover [TOR, 12a].

\subsection{Les différentes formes de proximité}

Selon l'école française de la proximité [BOU, 08a], la coordination entre les acteurs passe donc par l'articulation de deux formes de proximité : la proximité géographique qui renvoie à la séparation des agents dans l'espace, et la proximité organisée qui désigne la "capacité d'une organisation à faire interagir ses membres ", l'organisation en question pouvant être une firme, un réseau, etc. [RAL, 04]. La coordination entre acteurs repose ainsi sur d'autres facteurs que la seule co-localisation, la proximité organisée se référant aux normes collectives et codes communs de communication qui stabilisent le système de relations et rendent la collaboration possible [TOR, 05, TOR, 10, ZIM, 08]. La notion de proximité organisée fait encore débat, les auteurs de l'école française de la proximité se rattachant à deux courants principaux : les interactionnistes [RAL, 04] et les institutionnalistes [TAL, 05, 08, 10, COL, 08]. 
Pour les tenants de l'approche interactionniste, il n'existe qu'une forme de proximité de type organisé, la proximité organisationnelle, mais qui peut se décliner en deux logiques d'appartenance et de similitude. La première logique implique que l'appartenance à une même organisation a pour conséquence de faciliter les coopérations entre ses membres compte tenu des règles et des routines sur lesquelles ils fondent leur comportement. La logique de similitude présuppose que les membres d'une entité économique partagent le même système de représentations et de connaissances, améliorant de ce fait leur capacité à interagir [RAL, 04, TOR, 05].

Pour les institutionnalistes, il existe deux formes de proximité non spatiale, la proximité organisationnelle et la proximité institutionnelle. La proximité organisationnelle renvoie aux ressources complémentaires détenues par des acteurs au sein d'une même organisation (groupe, ...) ou ensemble d'organisations (réseau de coopération, secteur, système productif local, ...). La proximité institutionnelle repose quant à elle sur l'adhésion des acteurs à des règles d'action communes, formelles et informelles et à un système commun de représentations et de valeurs [GIL, 05, TAL, 05]. Celle-ci met en exergue le fait que ces règles communes et institutions garantes de la coordination, ne sont pas immuables et résultent de compromis établis entre acteurs, porteurs d'intérêts divergents et potentiellement conflictuels. Il est possible toutefois de réconcilier les tenants des deux courants si nous considérons que la logique d'appartenance peut se référer à la proximité organisationnelle et la logique de similitude à la proximité institutionnelle, sans évacuer la dimension sociale et politique des modes de coordination entre acteurs [CAR,08]. La proximité organisationnelle peut être également conçue comme une forme particulière de proximité institutionnelle si on distingue de façon purement analytique la coordination cognitive de la coordination politique [TAL, 08].

Le courant de géographie économique évolutionniste ou évolutionnaire (pour une revue complète de ce courant, voir le Handbook of Evolutionary Economic Geography édité par Martin et Boschma en 2010 [MAR, 10]) a étendu ces formes de proximité en distinguant les différentes proximités suivantes : proximité organisationnelle, institutionnelle, géographique mais aussi proximité sociale et proximité cognitive [BOS, 04, 05]. L'objectif de ces analyses récentes est de proposer une approche dynamique des réseaux et de leur évolution spatiale, et de voir de quelle manière ces évolutions, qui dépendent des proximités en jeu, affectent à leur tour les différentes formes de proximité.

La proximité cognitive se réfère au fait que les personnes vont partager la même base de connaissances et de compétences et apprendre les unes des autres. Deux organisations seront cognitivement proches lorsqu'elles auront accumulé des connaissances similaires qui les rendront capables d'absorber de nouvelles connaissances produites par l'autre [NOO, 00, VIC, 16]. La proximité organisationnelle mesure le degré de similitude ou de partage des acteurs dans les arrangements organisationnels (à l'intérieur d'une organisation ou entre deux organisations). Selon Boschma [BOS, 04], il existe des degrés de proximité organisationnelle différents. Il y aurait ainsi un continuum de relations de proximité, qui vont d'une proximité organisationnelle faible entre les acteurs (marché au comptant) à une proximité forte à l'intérieur d'une entreprise ou d'un réseau organisé hiérarchiquement. Boschma relie cette différence de degré de proximité organisationnelle au degré d'autonomie des acteurs dans les relations (c'est-à-dire au fait par exemple que l'entreprise soit indépendante ou appartienne à un groupe). En s'appuyant sur les travaux consacrés à «l'encastrement » des acteurs [POL, 44, GRA, 85], Boschma définit la proximité sociale en termes de relations socialement encastrées à un niveau microéconomique, impliquant une confiance fondée sur l'amitié, les liens familiaux et l'expérience. Les structures amicales, les structures locales du travail ou les réseaux issus de collaborations passées s'appuient sur cette proximité sociale. Par contre la proximité sociale n'inclut pas les relations entre personnes partageant un ensemble de valeurs, ethniques ou religieuses, qui relèvent, au niveau macro, de la proximité institutionnelle [BOS, 04]. Enfin, la proximité institutionnelle se réfère à un encastrement des organisations dans des systèmes d'habitudes, de règles, de normes formelles et informelles, qui fixent les règles du jeu et un cadre incitatif et contraignant aux actions des organisations et à leurs collaborations [VIC, 16]. 
Les différentes formes de proximités peuvent se substituer à la proximité géographique pour faciliter interactions, échanges et création de connaissances. Dans une perspective de long terme des réseaux d'innovation, est pris en compte la fait que les proximités puissent se renforcer au fil des années du fait de liens de collaboration antérieurs [BAL, 15].

\section{La complémentarité des coopérations locales et à distance}

Les différentes formes de proximité peuvent s'avérer problématiques si elles conduisent à une dépendance de sentier des réseaux d'innovation et dans certains cas, à un verrouillage régional (les termes de "path dependence" et de "lock-in" étant empruntés au Darwinisme et à la théorie évolutionniste de l'innovation). Dans cette optique, certains regroupements d'acteurs peuvent se retrouver «piégés» dans une dynamique d'interactions négatives, s'ils privilégient de manière systématique les interactions locales au détriment de l'ouverture sur l'extérieur [BOS, 04, 05, BRO, 08]. Cette logique peut à terme menacer la pérennité des entreprises qui éprouveront des difficultés à modifier leur trajectoire et à intégrer de nouveaux entrants, entreprises ou scientifiques.

Comme l'illustre le tableau suivant, toutes les formes de proximités peuvent être sources d'enfermement et synonyme d'inertie, de fonctionnement bureaucratique, de manque d'ouverture à des connaissances extra territoriales.

\begin{tabular}{|c|c|c|c|c|}
\hline Proximity & Key dimension & Too little proximity & $\begin{array}{l}\text { Too much } \\
\text { proximity }\end{array}$ & Possible solutions \\
\hline Cognitive & Knowledge gap & Misunderstanding & $\begin{array}{c}\text { Lack of sources of } \\
\text { novelty }\end{array}$ & $\begin{array}{l}\text { Common knowledge } \\
\text { base with diverse but } \\
\text { complementary } \\
\text { capabilities }\end{array}$ \\
\hline Organizational & Control & Opportunism & Bureaucracy & $\begin{array}{c}\text { Loosely coupled } \\
\text { system }\end{array}$ \\
\hline Social & $\begin{array}{l}\text { Trust (based on } \\
\text { social relations) }\end{array}$ & Opportunism & $\begin{array}{l}\text { No economic } \\
\text { rationale }\end{array}$ & $\begin{array}{l}\text { Mixture of embedded } \\
\text { and market relations }\end{array}$ \\
\hline Institutional & $\begin{array}{c}\text { Trust (based on } \\
\text { common institutions) }\end{array}$ & Opportunism & Lock-in and inertia & $\begin{array}{c}\text { Institutional checks } \\
\text { and balances }\end{array}$ \\
\hline Geographical & Distance & $\begin{array}{l}\text { No spatial } \\
\text { externalities }\end{array}$ & $\begin{array}{c}\text { Lack of } \\
\text { geographical } \\
\text { openess }\end{array}$ & $\begin{array}{c}\text { Mix of local "buzz" } \\
\text { and extra-local } \\
\text { linkages }\end{array}$ \\
\hline
\end{tabular}

Tableau 1. Les cinq formes de proximités (source : Boschma (2005) [BOS, 05])

Pour que des innovations voient le jour, il est donc important que ces connaissances encastrées localement puissent être combinées avec des connaissances extérieures. Cela exige de la part des entreprises des liens externes avec des acteurs qui peuvent être quelquefois éloignés de leur propre base de connaissances [TER, 11, PAR, 12]. À ce titre, si les entreprises ont besoin de ces contacts en face à face et de ces informations et connaissances échangées dans une même industrie et dans une même région ou territoire (le buzz), elles auront également besoin de pipelines, c'est-à-dire de canaux de transmission de connaissances plus distantes [BAT, 04]. Le buzz encourage le développement de valeurs partagées, de schémas d'interprétation communs typiques des communautés de pratiques [WEN, 98, AMI, 08] et il permet aux acteurs, pour certains localisés à l'extérieur de la région, de s'engager dans des apprentissages et résolutions de problèmes. Les connaissances transmises via les pipelines offrent la possibilité d'innover de façon plus radicale. Cependant, l'établissement de ces pipelines est un processus complexe et coûteux et il exige des investissements et ressources plus facilement disponibles dans les entreprises de grande taille. 
De même, contrairement à la thèse d'une supériorité de certains territoires comme les zones urbaines pour innover [HUR, 09, MCC, 07], certains auteurs affirment que seules les firmes dont l'activité d'innovation nécessite d'intenses et fréquentes innovations ont un intérêt à se localiser dans des aires métropolitaines [SHE, 12]. Dans son analyse du lien espace-innovation au Québec, Shearmur [SHE, 11] (2011) montre que ce besoin de proximité avec les villes varie en fonction du type d'innovations en jeu. Pour les innovations de procédés, la distance aux aires urbaines s'avère être un déterminant majeur de l'innovation alors que ce résultat est moins net en ce qui concerne les innovations de produits. Si les connaissances, les réseaux et les ressources intangibles sont des ingrédients des processus d'innovation, les infrastructures physiques et services associés (moyens de transport, hôtels, etc.) sont aussi requis. Ce sont en effet les garants de cette accessibilité aux points d'entrée des pipelines et, par conséquent, aux connaissances et collaborations externes. Les entreprises peuvent également compenser de faibles ressources externes en développant leurs ressources internes pour innover, en particulier, la capacité de recherche et développement, la qualification de leur personnel ou l'utilisation des TIC [GAL, 06].

Par ailleurs, la sélection des partenaires devra prendre en compte le fait que la distance cognitive $[\mathrm{NOO}, 00]$ ne doit pas être trop forte. C'est en effet nécessaire pour que les connaissances externes puissent être «absorbées » et donner lieu, en interne, à de nouveaux développements. En réalité, il existerait une distance cognitive optimale : elle ne doit certes pas être trop importante pour faciliter les échanges et la compréhension mutuelle, mais pas non plus trop faible sous peine de ne pas donner lieu à des développements qui soient réellement nouveaux pour les entreprises [NOO, 07]. Doloreux et Shearmur [DOL, 11] (2011) ajoutent que la qualité et la fréquence des collaborations sont certainement des variables déterminantes dans la réussite des projets collaboratifs.

Prenant à contrepied la thèse du «tout local », différents travaux montrent que les collaborations opèrent plutôt aux niveaux nationaux et internationaux [MAC, 03, FRE, 03, BAT, 04, DOL, 04, MCC, 07, MCC, 07, BOS, 05]. Ainsi les entreprises agroalimentaires combinent-elles par exemple différentes proximités pour gérer leurs partenariats, et elles ne se contentent pas de coopérer avec des partenaires géographiquement proches [TAN, 15]. L'analyse des coopérations pour innover des entreprises agroalimentaires témoigne du fait que le choix d'un partenaire pour innover est surtout dicté par des considérations d'ordre organisationnel (lintégration dans un groupe en particulier) ou stratégique. L'appartenance à un groupe constitue un moyen pour les entreprises, quelle que soit leur localisation, d'accéder aux ressources du groupe ainsi qu'à ses réseaux de coopération qui sont le plus souvent établis au niveau national et international [GAL, 11].

\section{En conclusion - Territoires de projet et nouveaux modes de gouvernance}

En réalité, les exemples de clusters qui fonctionnent selon le modèle idéal combinant la proximité géographique et la proximité organisée sont assez peu nombreux. La Silicon Valley reste emblématique de ce mode d'organisation, mais peu de régions ont réussi à répliquer avec succès ce modèle. Comme nous l'avons mentionné, il existe sans aucun doute des effets de localisation, ne serait-ce que parce que les réseaux interpersonnels et la mobilité de la main d'œuvre sont généralement circonscrits dans le cadre d'un territoire donné [BRE, 01]. La proximité géographique constatée dans ces regroupements d'acteurs proviendrait de l'encastrement des relations et d'une proximité relationnelle [GRO, 02, TOR, 05, AMI, 08] alors que les réseaux sont par définition a-spatiaux [BOS, 05, TER, 11].

Actuellement, la transition écologique ou techno-écologique [PIC, 16] remet profondément en question les fondements mêmes de nos sociétés. Les dernières années ont vu une remise en cause croissante des approches «technologisantes » des processus d'innovation pour promouvoir, au sein des territoires, un objectif de développement durable dans toutes ses dimensions, aussi bien économique qu'environnementale [DJE, 09] ou sociale [CRA, 16]. En effet, dépassant le simple verdissement du modèle actuel de société, la transition écologique repose en particulier sur l'innovation sociale et sociétale et l'émergence de nouvelles gouvernances, de nouvelles manières d'agir et de produire, de 
nouvelles pratiques de consommation plus vertueuses qui soient construites et partagées par l'ensemble des acteurs pour constituer progressivement de nouvelles références collectives. Ainsi, l'innovation sociale a fait l'objet d'une littérature foisonnante, et recouvre des définitions et réalités diverses [VAN, 16, RIC, 12a].

Les auteurs insistent généralement sur la dimension participative de l'émergence et du développement de l'innovation sociale, dimension collective qui s'appuie généralement sur les réseaux et la coordination d'acteurs dans les territoires [RIC, 12b]. L'innovation sociale est ainsi généralement associée à des initiatives locales, suivant une logique de type bottom-up [HAR, 06, MOU, 07].

La question se pose de la capacité de résilience des territoires et des sociétés [HOP, 10]. Il s'agit alors de mettre en œuvre un développement territorial durable, soit un mode de développement qui, à l'échelle du territoire, prend en compte les dimensions économiques, sociales et environnementales [GAL, 16]. Les territoires sont en effet en première ligne dans la mesure où ces innovations naissent et sont activées grâce à des initiatives locales et territorialisées de la transformation de nos sociétés, et qu'elles nécessitent de nouvelles formes de gouvernance. En effet, les différentes parties prenantes doivent être capables de concilier des intérêts qui peuvent être distincts pour construire un projet collectif [TOR, 12b], projet qui résulte donc de compromis provisoires entre des acteurs privés et publics, aux intérêts divergents et parfois contradictoires [GIL, 05]. Dans cette perspective, la politique publique occupe une place centrale dans ce dispositif en jouant un rôle de coordinateur à différentes échelles territoriales et d'activation des différentes proximités.

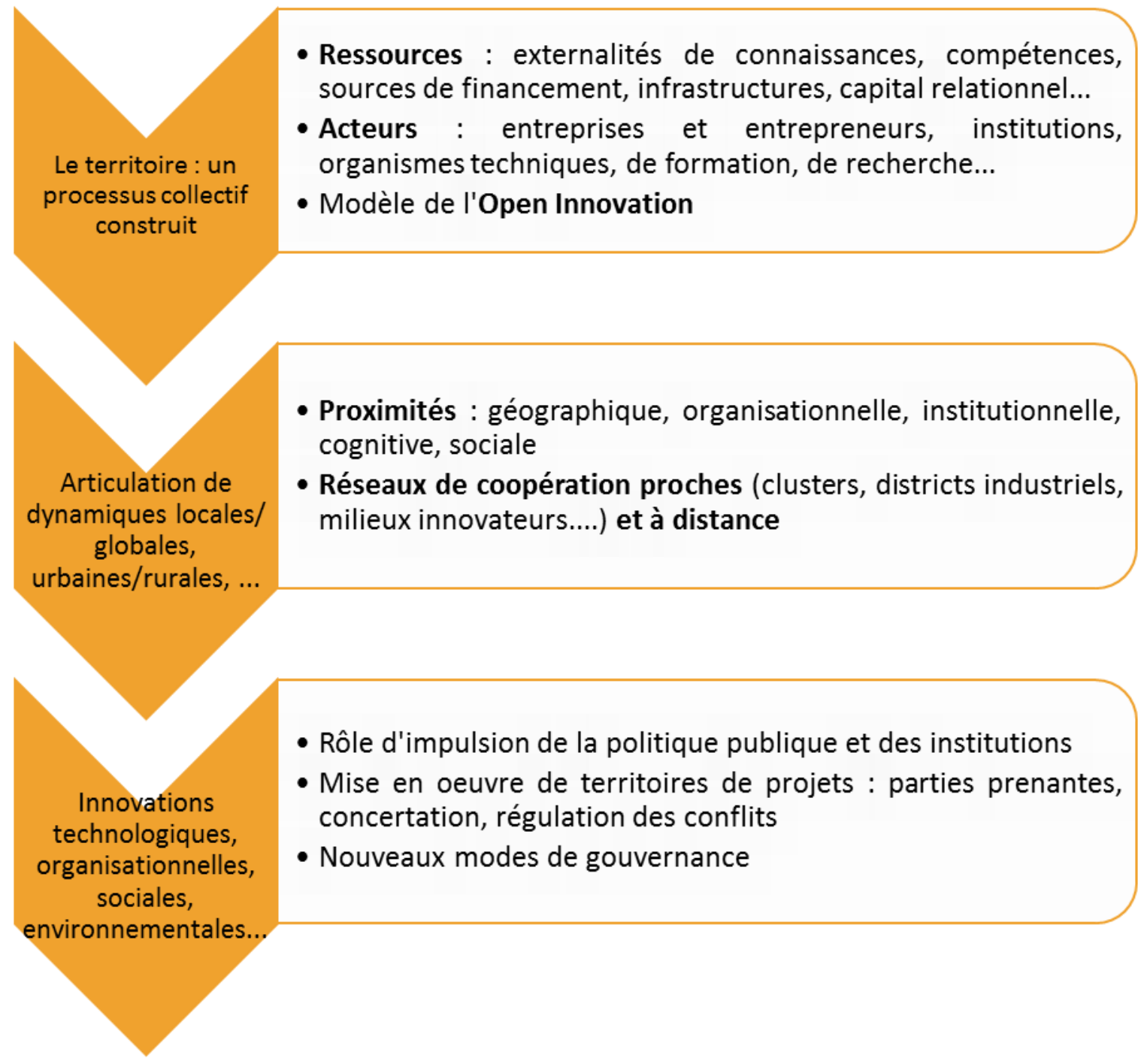




\section{Bibliographie}

[AMI, 08] AMIN A., RoBerTs J., "Knowing in action: Beyond communities of practice”, Research Policy, 37, p. 353-369, 2008.

[AUD, 96] AUdRETSCH D.B., FELDMAN M.P., "R\&D spillovers and the geography of innovation and production", American Economic Review, 86 (4), p. 253-273, 1996.

[AYD, 86] AYDALOT P. (dir.), Milieux innovateurs en Europe, GREMI, Paris, 1986.

[BAL, 15] BALland P.A., Boschma R., FrenKen K., "Proximity and innovation: From statics to dynamics", Regional Studies, 49 (6), p. 907-920, 2015.

[BAT, 04] Bathelt H., Malmberg A., Maskell P., "Clusters and knowledge: local buzz, global pipelines and the process of knowledge creation", Progress in Human Geography, 28 (1), p. 31-56, 2004.

[BAT, 08] BATHELT H., ShULDT N., "Between luminaires and meat grinders: International trade fairs as temporary clusters", Regional Studies, 42 (6), p. 853-868, 2008.

[BEC, 79] BECATTINI G., "Dal settore industriale al distretto industriale: alcune considerazioni sull'unità di indagine della politica industriale", Rivista di economia e politica industriale, 2, p. 7-21, 1979.

[BEC, 90] BeCATTINI G., "The Marshallian Industrial Districts as a Socio-Economic Notion”, in F. PyKE, G. BeCATTINI, W. SENGENBERGER (eds.), Industrial Districts and Inter-Firm Cooperation in Italy, International Institute of Labour Studies, Genève, 1990.

[BEN, 13] BEN LeTAIFA S., RABEAU Y., "Too close to collaborate? How geographic proximity could impede entrepreneurship and innovation”, Journal of Business Research, 66 (10), p. 2071-2078, 2013.

[BOS, 04] Boschma R., « Proximité et innovation, Économie rurale », 280 (1), p. 8-24, 2004.

[BOS, 05] Boschma R., "Proximity and innovation: a critical assessment", Regional Studies, 39 (1), p. 61-74, 2005.

[BOU, 08a] Bouba-Olga O., CARRINCAZEAUX C., CORIS C., « La proximité, 15 ans déjà ! Première partie : propositions théoriques », Revue d'Économie Régionale et Urbaine, 3, p. 279-287, 2008.

[BOU, 08b] BoubA-Olga O., Grosseti M., « Socio-économie de proximité », Revue d'Économie Régionale et Urbaine, 3, p. 311-328, 2008.

[BRE, 01] BRESCHI S., LISSONI F., "Knowledge spillovers and local innovation systems: a critical survey", Industrial and Corporate Change, 10 (4), p. 975-1005, 2001.

[BRO, 08] BRoekel T., MEder A., "The Bright and Dark Side of cooperation for regional innovation Performance", Papers in Evolutionary Economic Geography, 08.11, Utrecht University, 2008.

[BRU, 82] BRUSCO S., "The Emilian model: productive decentralisation and social integration", Cambridge Journal of Economics, 6, p. 167-184, 1982.

[CAM, 06] CAmagni R., Maillat D. (eds.), Milieux innovateurs - Théorie et politiques, Economica Anthropos, Paris, $450 \mathrm{p}, 2006$.

[CAM, 95] CAMAGni R., Espace et temps dans le concept de milieu innovateur, in A. RALlET., A. ToRre (dir.) Économie industrielle et économie spatiale, Economica, Paris, p. 193-210, 1995.

[CAR, 08] CARRINCAZEAUX C., LUNG Y., ViCENTE J., "The scientific trajectory of the French school of proximity: interaction-and institution-based approaches to regional innovation systems", European Planning Studies, 16 (5), p. 617-628, 2008.

[CHA, 12] Chalaye S., MASSARD N., Géographie de l'innovation en Europe - Observer la diversité des régions françaises, Travaux nº 15, DATAR, La Documentation Française, Paris, 2012.

[CHE, 03] CHESBROUGH H., Open Innovation - the new imperative for creating and profiting from technology, Harvard Business School Press, 2003.

[CMI, 15] CMI, Innovation et territoires de faible densité, Étude conduite pour le compte du Commissariat général à l'égalité des territoires, Juin, 134 p., 2015.

[COH, 89] COHEN W.M., LEVINTHAL D.A., "Innovation and learning: the two faces of R\&D", Economic Journal, 99, September, p. 569-596, 1989. 
[COH, 90] COHEN W.M., LEVINTHAL D.A., "Absorptive capacity: a new perspective on learning and innovation", Administrative Science Quarterly, 35, p. 128-152, 1990.

[COL, 04] Colletis G., PeCQueur B., Révélation de ressources spécifiques et coordination située », Quatrièmes Journées de la proximité, Marseille 17-18 juin, 2004.

[COL, 08] COLLETIS-WAHL K., «Micro-institutions et proximités : quelles lectures des dynamiques territoriales ?», Revue d'Économie Régionale \& Urbaine, (2), p. 251-264, 2008.

[COM, 03] COMPTE C., «Enjeux de la formation ouverte et à distance : technologies et apprentissages », dans I. SALEH, D. LEPAGE et S. BOUYAHI (dir.), Les TIC au cour de l'enseignement à distance, Actes Huit Coll., Université de Paris VIII, 2003.

[CRA, 16] CRAWFORD M.B., Contact : pourquoi nous avons perdu le monde, et comment le retrouver, La Découverte, Paris, 2016.

[DEP, 09] DEPRET M.H., HAMDOUCH A., « Clusters, réseaux d'innovation et dynamiques de proximité dans les secteurs high-tech : une revue critique de la littérature récente », Revue d'Économie Industrielle, 128 (4), p. 21-53, 2009.

[DJE, 09] DJellal F., Gallouj F., «Innovation dans les services et entrepreneuriat: au-delà des conceptions industrialistes et technologistes du développement durable », Innovations, 29(1), p. 59-86, 2009.

[DOL, 04] DoloreuX D., "Regional innovation systems in Canada: a comparative survey", Regional Studies, 38, p. 481494, 2004.

[DOL, 11] DolOREUX D., SHEARMUR R., "Collaboration, information and the geography of innovation in knowledge intensive business services", Journal of Economic Geography, 12 (1), p. 79-105, 2011.

[FOR, 00] FORAY D., L'économie de la connaissance, La Découverte, Paris, 2000.

[FRE, 03] FREEL M.S., "Sectoral patterns of small firm innovation, networking and proximity", Research Policy, 32, p. 751-770, 2003.

[GAL, 04] Gallaud D., TORRE A., "Geographical proximity and the diffusion of knowledge: the case of SME's in biotechnology”, in G. FUCHS, P. SHAPIRA, A. KOCH (eds.), Rethinking Regional Innovation, Springer, USA, 2004.

[GAl, 06] Galliano D., Roux P., Soulie N., «Dynamiques d'adoption des TIC et densité des espaces. Quelles spécificités pour les firmes rurales françaises? », Économie Rurale, 3, p. 4-19, 2006.

[GAL, 11] GALLIANO D., GAREDEW L., MAGRINI M.B., «Les déterminants organisationnels de l'innovation-produit : les spécificités des firmes agroalimentaires françaises », in DGCIS, L'innovation dans les entreprises : moteurs, moyens et enjeux, DGCIS Éditions, p. 50-68, 2011.

[GAL, 16] GALLAUD D., LAPERCHE B., Économie circulaire et développement durable - Écologie industrielle et circuit courts, Iste Éditions, London, 2016.

[GAR, 92] GAROFOLI G., «Les systèmes de petites entreprises : un cas paradigmatique du développement endogène », in G. BENKo, A. LiPIETZ (eds.), Les régions qui gagnent. Districts et réseaux : Les nouveaux paradigmes de la géographie économique, PUF, Paris, 1992.

[GIL, 05] GiLly J.P., LuNG Y., « Proximités, secteurs et territoires », Cahiers du GRES, 9, Mai, 20 p., 2005.

[GOD, 10] Godet M., DuRAnCe P., Mousli M., Créativité et innovation dans les territoires, Rapport, Conseil d'Analyse Économique, 465 p. (http://www.ladocumentationfrancaise.fr/var/storage/rapports-publics/104000479.pdf), 2010.

[GOR, 05] GORdON I., MCCANN P., "Innovation, agglomeration and regional development", Journal of Economic Geography, 5 (5), p. 523-543, 2005.

[GRA, 85] GRANOVETTER M., "Economic action and social structure. The problem of embeddedness", American Journal of Sociology, 91 (3), p. 481-510, 1985.

[GRO, 02] GRossetTI M., BÈs M.P., "Proximité spatiale et relations science - industrie : savoirs tacites ou encastrement (Polanyi ou Polanyi) ? Réflexions à partir d'une étude sur les collaborations CNRS - entreprises en France », Revue d'Économie Régionale et Urbaine, 5, p. 777-788, 2002.

[GRO, 03] GrossetTi M., Bes M.P., « Dynamiques des réseaux et des cercles. Encastrements et découplages », Revue d'économie industrielle, 103 (1), p. 43-58, 2003.

[HAR, 06] HARRISSON D., VEZINA M., «L'innovation sociale : une introduction », Annals of Public and Cooperative Economics 77(2), p. 129-138, 2006. 
[HOP, 10] HoPKINS R., Manuel de transition : de la dépendance au pétrole à la résilience locale, Éditions Ecosociété, 2010.

[HUR, 09] HuRiot J.M., BouRdEAU-LEPAGE L., Économie des villes contemporaines, Economica, Paris, 2009.

[KAR, 05] KARLSSON C., JohansSON B., StOUGH R., Industrial Clusters and Inter-Firm Networks, Edward Elgar, London, 2005.

[KLE, 00] KLEIN O., «Des districts industriels à la «proximité » : l'analyse critique de la communication au-delà de la métaphore spatiale ?», Revue d'Économie Régionale et Urbaine, 2, p. 281-300, 2000.

[KNO, 06] KNOBEn J., OeRlemans L.A., "Proximity and inter-organizational collaboration: A literature review", International Journal of Management Reviews, 8 (2), p. 71-89, 2006.

[KRU, 91] KRUGMAN P., Geography and Trade, MIT Press, Cambridge, 1991.

[LUN, 05] LUNDVALL B.A., "National innovation systems - analytical concept and development tool", $10^{\text {th }}$ Danish Research Unit for Industrial Dynamics (DRUID) Conference, Copenhagen, Denmark [en ligne] (http://www.druid.dk/conferences/summer2005/papers/ds2005-603.pdf), 2005.

[LUN, 92] Lundvall B.A. (ed.), National systems of innovation. Towards a theory of innovation and interactive learning, Editions Pinter Publishers, London, 1992.

[MAC, 03] MAC Kelvey M., Alm H., RicCABONi M., "Does co-location matter for formal collaboration in the Swedish biotechnology pharmaceutical sector?”, Research policy, 1394, p. 1-19, 2003.

[MAD, 08] MADIES T., PRAGER J.C., Innovation et compétitivité des régions, Rapport La Documentation française. Paris, 394 p. (http://www.ladocumentationfrancaise.fr/var/storage/rapports-publics/084000589.pdf), 2008.

[MAI, 95] MAILlat, D., Milieux innovateurs et dynamiques territoriales. in A. RALLET, A. TORRE (eds.), Économie industrielle et économie spatiale, Economica, Paris, p. 211-233, 1995.

[MAL, 02] MALmberg A., MASKell P., "The Elusive Concept of Localization Economies: Towards a Knowledge-Based Theory of Spatial Clustering", Environment and Planning, 34 (3), p. 429-449, 2002.

[MAR, 10] MARTIN R., Boschma R. (eds.), The Handbook of Evolutionary Economic Geography, Edward Elgar, 559 p., 2010.

[MAR, 19] MARShall A., Industry and Trade, Mac Millan, Londres, 875 p., 1919.

[MAS, 01] MASSARD N., «Externalités de connaissances et géographie de l'innovation : les enseignements des études empiriques », Séminaire CGP «La place de la France dans l'économie des connaissances », 23 p., 2001.

[MAS, 05] MASKell P., BAthelt H., Malmberg A., "Building global knowledge pipelines: the role of temporary clusters", DRUID (Danish Research Unit for Industrial Dynamics) Working Paper 05-20, 17 p., 2005.

[MCC, 07] MCCANN P., "Sketching Out a Model of Innovation, Face-to-Face Interaction and Economic Geography", Spatial Economic Analysis, 2 (2), p. 117-134, 2007.

[MEN, 06] MENDEZ A., MERCIER D., « Compétences-clés de territoires. Le rôle des relations inter-organisationnelles », Revue Française de Gestion, 5 (164), p. 253- 275, 2006.

[MOR, 98] MORGAN K., COOKE P., The associational economy: firms, regions, and innovation, Oxford University Press, Oxford, 1998.

[MOU, 07] Moulaert F., Martinelli F., GonZÁlez S., Swyngedouw E., "Introduction: Social Innovation and Governance in European Cities: Urban Development Between Path Dependency and Radical Innovation", European Urban and Regional Studies, 14(3), p. 195-209, 2007.

[NEL, 93] NELSON R., National Innovation Systems: a Comparative Analysis, Oxford University Press, New York, 1993.

[NOO, 00] NоOTEBOOM B., "Learning by interaction: absorptive capacity, cognitive distance and governance", Journal of management and governance, 4 (1-2), p. 69-92, 2000.

[NOO, 07] Nooteboom B., Van HaverbeKe W., Duysters G., Gilsing V., VAn Den Oord A., “Optimal cognitive distance and absorptive capacity", Research policy, 36 (7), p. 1016-1034, 2007.

[OUR, 15] OURAL A., L'innovation au pouvoir! Pour une action publique réinventée au service des Territoires, Rapport établi avec l'appui du secrétariat général pour la modernisation de l'action publique, Avril, $110 \mathrm{p}$. (http://www.modernisation.gouv.fr/sites/default/files/fichiers-attaches/innovation_territoriale-rapport-2015-04.pdf), 2015. 
[PAR, 12] Parrilli M.D., Curbelo J.L., Cooke P., "Introduction”, in M.D. PARrilli., J.L. Curbelo, P. CoOKe (eds.), Innovation, Global Change and territorial resilience, Edward Elgar, Cheltenham, UK, 2012.

[PER, 64] PeRrouX F., L’Économie du XX'e siècle, Presses Universitaires de France, Paris, 1964.

[PIC, 16] PICARD F., TANGUY C., Innovations et transition techno-écologique, Éditions Iste, London, 2016.

[POL, 44] POLANYI K., The great transformation, Beacon Press, Boston, 1944.

[POR, 03] PORTER M.E., “The economic performance of regions”, Regional Studies, 37 (6 et 7), p. 549-579, 2003.

[POR, 98] PORTER M.E., "Clusters and competition: new agendas for companies, governments and institutions", in M.E. PORTER, On Competition, Harvard Business School Press, Boston, MA, 1998.

[RAL, 04] RAllet A., TORRE A., « Proximité et localisation », Économie Rurale, 280, p. 25-41, 2004.

[RAL, 07] RALLET A, TORRE A., Quelles proximités pour innover ?, L’Harmattan, Paris, 2007.

[RER, 08] RERU, « La proximité : 15 ans déjà ! », 3, p. 279-498, 2008.

[RER, 93] RERU, Économie des proximités, 3, 1993.

[RIC, 12a] Richez-BatTesti N., Petrella F., Vallade D., «L'innovation sociale, une notion aux usages pluriels : Quels enjeux et défis pour l'analyse?», Innovations, 38(2), p. 15-36, 2012.

[RIC, 12b] RICHEZ-BATTESTI N., VALLADE D., «Éditorial. Innovation sociale, normalisation et régulation », Innovations, (38), p. 5-13, 2012.

[SCH, 14] SCHAEFER D., Débrider l'innovation : enjeux pour les entreprises et l'emploi, défi pour les politiques publiques, Rapport au nom de la Commission Économie et Croissance et du Développement Économique Régional, Novembre, 81 p., 2014.

[SHE, 11] SHEARmuR R., "Innovation, Regions and Proximity: From Neo-Regionalism to Spatial Analysis", Regional Studies, 45 (9), p. 1225-1243, 2011.

[SHE, 12] SHEARMUR R., "Are cities the font of innovation? A critical review of the literature on cities and innovation", Cities, 29, p. S9-S18, 2012.

[SUI, 08] SUIRE S., VICENTE J., «Théorie économique des clusters et management des réseaux d'entreprises innovantes », Revue Française de Gestion, 184, p. 119-136, 2008.

[TAL, 05] TALBOT D., «Une compréhension institutionnaliste de la proximité organisationnelle : le cas d'EADS », Cahier du GRES, 22, 20 p., 2005.

[TAL, 08] TALBOT D., «Les institutions créatrices de proximités », Revue d'Économie Régionale et Urbaine, 3, p. 289$310,2008$.

[TAL, 10] TALBOT, D., «La dimension politique dans l'approche de la proximité », Géographie, économie, société, 12 (2), p. 125-144, 2010.

[TAN, 15] TANGUY C., Gallaud D., MARTin M., Reboud S., « Quelle est la propension des entreprises à coopérer pour innover et à quelles échelles spatiales ? Une analyse des entreprises agroalimentaires françaises sur la période 20062008 », Revue d'Économie Régionale et Urbaine, 3, p. 453-479, 2015.

[TAN, 16] TANGUY C., UZUNIDIS D., Milieu innovateur et entrepreneuriat : la force des proximités et des réseaux, Technologie et Innovation, 16 (1), 11 p. (http://www.openscience.fr/IMG/pdf/iste_techinn_v1n1_2.pdf), 2016.

[TER, 11] Ter Wal A., Boschma R., "Co-evolution of firms, industries and networks in space", Regional Studies, 45, p. 919-933, 2011.

[TOR, 05] TORRE A, Rallet A., "Proximity and Localization”, Regional Studies, 39(1), p. 47-59, 2005.

[TOR, 06] TORRE A., «Clusters et systèmes locaux d'innovation. Un retour critique sur les hypothèses naturalistes de la transmission des connaissances à l'aide des catégories de l'économie de la proximité », Régions et Développement, 24, p. 15-44, 2006.

[TOR, 10] TORRE A., « Jalons pour une analyse dynamique des Proximités », Revue d'Économie Régionale et Urbaine, 3, p. 409-437, 2010.

[TOR, 12a] TORRE A., « Géographie de l'innovation », Territoires 2040, 6, p. 53-61, 2012.

[TOR, 12b] TORRE A., BeuRET J.E., Proximités territoriales, Economica, Paris, 2012. 
[TOR, 14] TORRE A., TANGUY C., «Les systèmes territoriaux d'innovation : où en est-on ? », in S. BOUTILLIER, J. Forest, D. Gallaud, B. LAperche, C. TANGuY, L. TeMri (coord.), Principes d'Économie de l'Innovation, Bruxelles, Peter Lang, p. 307-319, 2014.

[TOR, 15] TORRE A., ZIMMERMANN J.B., «Des clusters aux écosystèmes industriels locaux », Revue d'Économie Industrielle, 152, p. 13-38, 2015.

[VAN, 16] VAN DER HAVE R. P., RUBALCABA L., "Social innovation research: An emerging area of innovation studies?", Research Policy, 45(9), p. 1923-1935, 2016.

[VEL, 93] VELTZ P., «D'une géographie des coûts à une géographie de l'organisation Quelques thèses sur l'évolution des rapports entreprises/territoires », Revue économique, 44 (4), p. 671-684, 1993.

[VEL, 94] Veltz P., Des territoires pour apprendre et innover, Éditions de l'Aube, Paris, 1994.

[VIC, 16] VICENTE J., Économie des clusters, La Découverte, Collection Repères, Paris, 126 p, 2016.

[WEN, 98] Wenger E., Communities of Practice: Learning, Meaning, and Identity, Cambridge University Press, 1998.

[ZIM ,98] ZIMMERMANN J.B., « Nomadisme et ancrage territorial : propositions méthodologiques pour l'analyse des relations firmes - territoires », Revue d'Économie Régionale et Urbaine, 2, p. 211-230, 1998.

[ZIM, 08] ZIMMERMANN J.B., «Le territoire dans l'analyse économique : Proximité géographique et proximité organisée », Revue Française de Gestion, 184, p. 105-118, 2008. 